\title{
Instantons And Baryon Mass Splittings in the MIT Bag Model
}

\author{
D. Klabučar \\ Physics Department of Zagreb University, P.O.B. 162, 41001 Zagreb, Croatia
}

\begin{abstract}
The contribution of instanton-induced effective inter-quark interactions to the baryon mass splittings was considered in the bag model. It is found that results are different from those obtained in the constituent quark model where the instanton effects are like those from one-gluon exchange. This is because in the context of the bag model calculation the one-body instanton-induced interaction has to be included.
\end{abstract}

12.38.Lg; 12.40.Aa; $14.20 .-\mathrm{c}$ 